\title{
Plastic Zone Analysis of Deep-Buried, Noncircular Tunnel and Application on the High-Speed Railway in the Karst Area
}

\author{
Hai Shi ${ }^{1}$ and Mingzhou Bai ${ }^{1,2}$ \\ ${ }^{1}$ School of Civil Engineering, Beijing Jiaotong University, Beijing, China \\ ${ }^{2}$ Beijing Key Laboratory of Track Engineering, Beijing, China \\ Correspondence should be addressed to Hai Shi; 516519566@qq.com
}

Received 16 January 2017; Revised 26 March 2017; Accepted 11 April 2017; Published 13 August 2017

Academic Editor: Francesco Marotti de Sciarra

Copyright (C) 2017 Hai Shi and Mingzhou Bai. This is an open access article distributed under the Creative Commons Attribution License, which permits unrestricted use, distribution, and reproduction in any medium, provided the original work is properly cited.

\begin{abstract}
With the conformal mapping function provided by Verruijt, the outland of a noncircular tunnel can be mapped to a circular unit in the complex plane and then spread the analytic function into a Laurent series. The stress unified solution of oval and horseshoe cross section can be determined using Muskhelishvili's complex variables function method. Subsequently, the solution can be taken into the Griffith strength failure criterion and determine the scale and shape of plastic zone in the tunnel surrounding rock. Aiming at the critical safety thickness between a concealed cave and tunnel in the karst area and determining whether the plastic zone of tunnel surrounding rock is connected with the plastic zone of cave as a judgment standard, the model of critical safety thickness among the concealed caves and tunnels is established. The numerical model is established in comparison with the computing method of rock plate critical safety thickness in actual engineering based on the Doumo tunnel engineering of Shanghai-Kunming (Guizhou segment) high-speed railway. The following conclusions can be drawn: the analytical approximation method has less indexes, and the output of this method is approximately close to actual engineering and numerical analysis, in which it is reliable and rational.
\end{abstract}

\section{Introduction}

The plastic zone range of the tunnel and the surrounding rock of underground caverns is the important base of determining its support and design. Both local and international studies on underground tunnels have led to abundant findings. The elastic-plastic problem of the plastic zone of the surrounding rock was proposed by Kastner [1]. Donald and Samir [2] modified the radius of plastic zone of the circular hole, which concluded the implicit expression. Yu and Zheng [3] revised the work of Kastner; Wang et al. [4] determined the shape and range of plastic zone of the surrounding rock based on the solution of the plastic zone with traditional elastic-plastic finite element method combined with adaptive mesh refinement method. According to energy theory of plasticity, the constitutive equations of nonlinear softening under uniaxial compression of the broad rock are promoted, and the research on the relationship between the equivalent stress and the strain relationship of the plastic zone of the underground caverns surrounding rock under various stress conditions is conducted based on unified strength theory $[5,6]$. Chen et al. [7] obtained the boundary plastic formula of the nonuniform field of roadway surrounding rock and predicted the size and range of the plastic zone of deep-buried underground tunnel under varying lateral pressure coefficients when considering the characteristics of the rock dilatancy and expansion effects and promoting the plastic zone of the surrounding rock of a circular tunnel under nonaxisymmetrical load; Sun and $\mathrm{Lu}$ [8] and Exadaktylos et al. [9] established the stress-strain softening model with the application of Mohr-Coulomb yield criterion and the elastic-plastic and softening-plastic residual based on bonded anchor reinforcement mechanism; Liu et al. [10] and Verruijt [11] obtained the formula in the plastic zone scope of deep-buried circular tunnel with the protection of the bolt after derivation. On the basis of the rock mechanical parameter measuring in situ stress field, modified equivalent radius elastic-plastic theory was adopted; Zha et al. [12] and Massinas and Sakellariou [13] calculated the size of the plastic zone of deep-buried roadway and analyzed the individual influence law of pressure coefficient and the coupling of the 


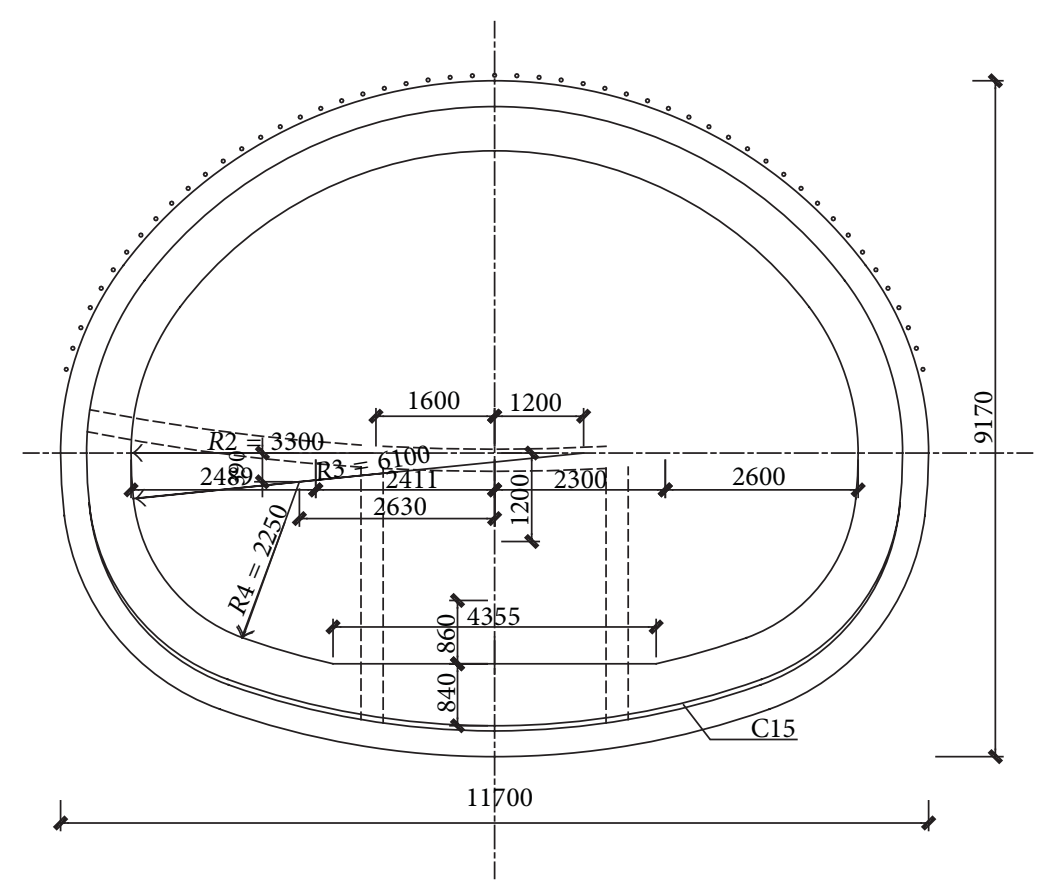

FIgURE 1: The geometry and related parameters of horseshoe-shaped tunnel.

plastic zone of roadway surrounding rock. Based on the abovementioned research on boundary conditions, the stress and displacement components of the circular underground chambers are easy to calculate. However, solving the stress function of the surrounding rock in a noncircular cavity perspective is extremely difficult [14-16]. Moreover, the literature in view of the range and the form of the noncircular tunnel plastic is relatively small, which provides an opportunity for this study.

During the process of highway railway tunnel construction in Southwestern China, the existence of geological disasters, such as karst, is an important factor affecting tunnel construction safety. The long-term hazards that concealed the karst cave have not been thoroughly solved because the influence factors of karst development are complicated, although a large number of karst problems have been dealt with. The construction of the karst tunnel has become a prominent problem whether the distance between concealed the karst cave and the tunnel is determined practical or not. In addition, a complete knowledge base and a systemic research have not been formed regarding the relationship between the karst cavity and the deformation and destruction characteristics. Considering the abovementioned problems, several qualitative and semiquantitative researches are carried out currently. The main methods are empirical analogy method, strength reduction theory $[17,18]$, supporting vector machine, and beam plate model analysis of catastrophe theory [19].

Therefore, according to the characteristics of deep-buried noncircular tunnel based on the abovementioned studies along with the help of conformal mapping regarding elasticplastic analysis of tunnel based on the mapping functions presented by Laurent series of infinite and simply connected domain, this article obtains a unified solution to the elasticplastic stress of the elliptical and horseshoe cross sections with the application of complex variable function submitted by Muskhelishvili and Radok [20]; furthermore, the study conveys the Griffith strength failure criterion to determine the size and shape of the plastic zone of the surrounding rock of underground tunnels. Moreover, it is used for the research on the tunnels of karst areas and the stability of concealed middle and small caves. To analyze and verify the feasibility and rationality of the methods, they are compared with the minimum thickness of the concealed middle and small caves determined in the actual engineering and the tunnel rock.

\section{Method for the Complex Variable Function of Deep-Buried Tunnel}

The effect of gravity gradient can not be considered at the time of the study because of the characteristics of deepburied depth and large diameter of the tunnel. According to the design standard of high-speed railway in China, the geometry and related parameters of horseshoe-shaped tunnel are shown in Figure 1. The infinite effect of external loads $P$ stands for the gravity to solve around the tunnel [21]. According to Muskhelishvili's complex variable function method and elastic-plastic mechanics theory, the lateral pressure coefficient is $\lambda$, the effect of external load from the infinite distance is $P$, and the depth of noncircular section tunnel is $H$. The surrounding rock stress after excavation is considered as holes, and plane strain problems of the stress points are around a semi-infinite plane. The calculation model is shown in Figure 2. 


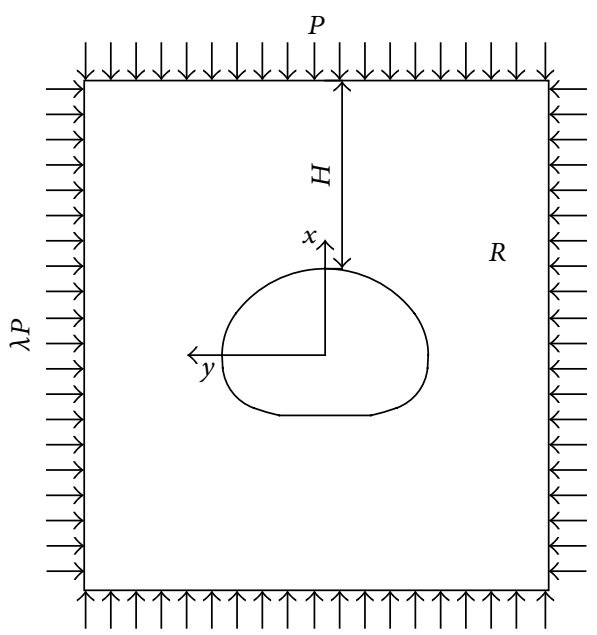

FIgure 2: Computational analysis model of deep tunnel complex variable method.

2.1. Basic Equations. The complex variable functions $\varphi_{1}(x)$ and $\psi_{1}(x)$ of stress in region $R$ are [22]

$$
\begin{aligned}
\sigma_{x}+\sigma_{y} & =4 \operatorname{Re}\left[\varphi_{1}^{\prime}(z)\right] \\
\sigma_{y}-\sigma_{x}+2 i \tau_{x y} & =2\left[\bar{z} \varphi_{1}^{\prime \prime}(z)+\psi_{1}^{\prime}(z)\right] .
\end{aligned}
$$

Then,

$$
\begin{aligned}
\sigma_{x} & =2 \operatorname{Re}\left[\varphi_{1}^{\prime}(z)\right]-\operatorname{Re}\left[\bar{z} \varphi_{1}^{\prime \prime}(z)+\psi_{1}^{\prime}(z)\right] \\
\sigma_{y} & =2 \operatorname{Re}\left[\varphi_{1}^{\prime}(z)\right]+\operatorname{Re}\left[\bar{z} \varphi_{1}^{\prime \prime}(z)+\psi_{1}^{\prime}(z)\right] \\
\tau_{x y} & =\operatorname{Im}\left[\bar{z} \varphi_{1}^{\prime \prime}(z)+\psi_{1}^{\prime}(z)\right],
\end{aligned}
$$

where $z$ is any point on the surrounding rock of the tunnel. $\sigma_{x}, \sigma_{y}, \tau_{x y}$ are the stress and strain components of any point. Functions $\varphi_{1}(x)$ and $\psi_{1}(x)$ under the bounded condition in the infinite point stress are analyzed. The expression is

$$
\begin{aligned}
\varphi_{1}(z)= & -\frac{1}{2 \pi(1+\kappa)}(X+i Y) \ln z+(B+i C) z \\
& +\varphi_{1}^{0}(z) \\
\psi_{1}(z)= & \frac{1}{2 \pi(1+\kappa)}(X-i Y) \ln z+\left(B^{\prime}+i C^{\prime}\right) z \\
& +\psi_{1}^{0}(z),
\end{aligned}
$$

where $B, B^{\prime}, C^{\prime}$ represent the depth of the tunnel, the bulk density of the surrounding rock, and the relationship of lateral pressure coefficient and surrounding rock stress, respectively. $\kappa$ is the positive integer. $\varphi_{1}^{0}(z)$ and $\psi_{1}^{0}(z)$ are the analytic functions of points in the neighborhood located at infinity, where $B=\left(\sigma_{x}^{\infty}+\sigma_{y}^{\infty}\right) / 4, B^{\prime}=\left(\sigma_{y}^{\infty}-\sigma_{x}^{\infty}\right) / 2, C^{\prime}=\tau_{x y}^{\infty}$, $\sigma_{x}^{\infty}, \sigma_{y}^{\infty}$, and $\tau_{x y}^{\infty}$ are the stress located at infinity.
2.2. Conformal Mapping. For noncircular section tunnel, $\varphi_{1}(x)$ and $\psi_{1}(x)$ are difficult to evaluate directly. In normal circumstances, the noncircular cross section tunnel in $z$ plane fault and the surfaces may be mapped to $\xi$ plane of the unit circle [23], and it is reflected in the form of Laurent series in the circle; that is,

$$
z=\omega(\xi)=R\left(\xi+\sum_{k=0}^{\infty} C_{k} \xi^{-k}\right)
$$

Any point on the plane $\xi$ can be expressed as $\xi$ in polar form; that is, $\xi=\rho e^{i \theta}=\rho(\cos \theta+i \sin \theta)$, where $R$ is the set of real numbers and can be expressed as the shape of the underground tunnel. $C_{k}$ is realistic when there is only one symmetry axis in the tunnel. According to the transformation formula, the stress amount transformation is

$$
\begin{aligned}
& \sigma_{\rho}+\sigma_{\theta}=\sigma_{x}+\sigma_{y}=4 \operatorname{Re}[\Phi(\xi)] \\
& \sigma_{\rho}-\sigma_{\theta}+2 i \tau_{\rho \theta}=\sigma_{x}-\sigma_{y}+2 i \tau_{x y} \\
& =\frac{2 \xi^{2}}{\rho^{2} \omega^{\prime}(\xi)}\left[\overline{\omega(\xi)} \Phi^{\prime}(\xi)+\omega^{\prime}(\xi) \Psi(z)\right],
\end{aligned}
$$

where Re is expressed separately in real and imaginary parts in the formula.

2.3. Stress Boundary Condition. Under the conformal mapping function, stress boundary conditions are available on $z$ plane and orthogonal curvilinear coordinates are expressed as a quantity of time and generally written as

$$
\varphi(\sigma)+\frac{\omega(\sigma)}{\overline{\omega^{\prime}(\sigma)}} \overline{\varphi^{\prime}(\sigma)}+\overline{\psi(\sigma)}=f(\sigma) .
$$

\section{Analysis of the Plastic Zone of Deep Oval-Shaped Cavern}

According to the design standard of high-speed railway in China, the geometry and related parameters of oval-shaped tunnel are shown in Figure 3. Suppose there is an oval-shaped tunnel located in $z$ plane, where $a$ is the semimajor axis and $b$ is the short half shaft. According to theory of conformal mapping, $z$ plane elliptic hole sections are mapped to $\xi$, the outlands on the unit circle of the plane.

The conformal mapping area is shown in Figure 4. Based on the abovementioned research, the mapping function expression [22] is

$$
z=\omega(\xi)=c\left(\xi+\frac{m}{\xi}\right)
$$

3.1. Analytical Functions for the Solution of $\varphi_{1}(x)$ and $\psi_{1}(x)$. Using (5) and $\sigma_{\theta}$ the analytic functions $\varphi_{1}(\xi)$ and $\psi_{1}(\xi)$ must be evaluated to obtain the stress components $\sigma_{\rho}$ and $\sigma_{\theta}$. Thus, 


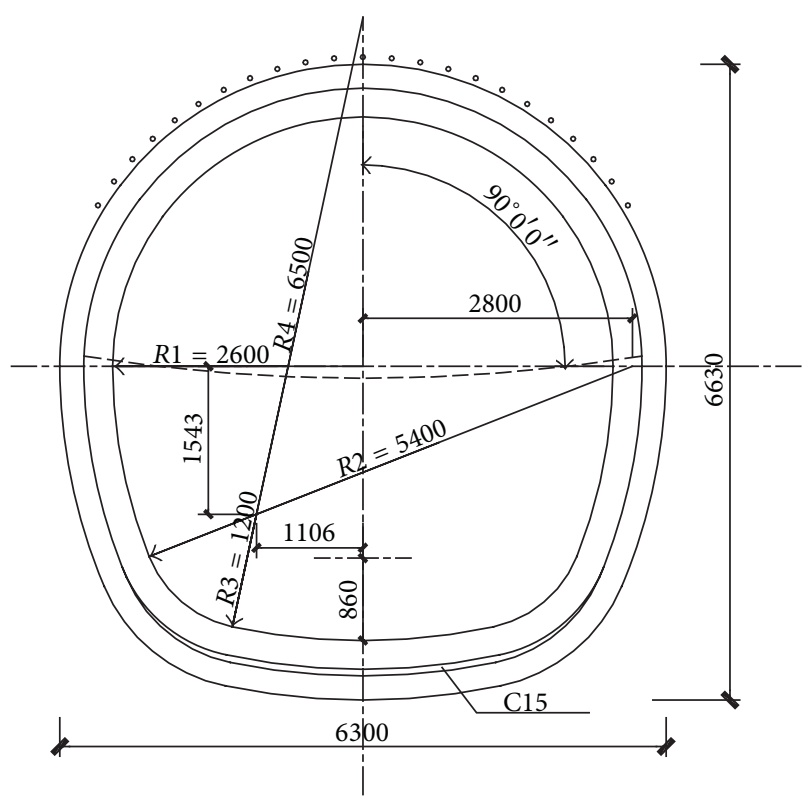

Figure 3: The geometry and related parameters of horseshoeshaped tunnel.

according to (3) representation of analytic functions, we do not consider the effect of edge load of the outer hole, $X=$ $Y=0$ [22], and it is based on the conformal mapping that

$$
\begin{aligned}
& \varphi_{1}(\xi)=B \omega+\varphi_{0}(\xi) \\
& \psi_{1}(\xi)=\left(B^{\prime}+i C^{\prime}\right) \omega+\psi_{0}(\xi) .
\end{aligned}
$$

This oval-shaped tunnel shows when it is at infinity, $B=$ $P(1+\lambda) / 4, B^{\prime}=P(1-\lambda) / 2, \tau_{x y}^{\infty}=0$. For analytic functions, the following should be calculated, $\varphi_{1}(\xi)$ and $\psi_{1}(\xi)$. On the edge of the hole in the tunnel, $\xi=\sigma$. Therefore, by using Formulas (8) and (4) into the boundary conditions (6), it will obtain

$$
\varphi(\sigma)+\frac{\omega(\sigma)}{\overline{\omega^{\prime}(\sigma)}} \overline{\varphi^{\prime}(\sigma)}+\overline{\psi(\sigma)}=-2 B \omega(\sigma)-B^{\prime} \overline{\omega(\sigma)}
$$

Perform Cauchy integral on both sides of Formula (9) by sorting to obtain $\varphi_{0}(\xi)$. Apply the conjugate results of both sides to obtain $\psi_{0}(\xi)$; that is,

$$
\begin{aligned}
\varphi_{0}(\xi)= & \frac{P c}{2 \xi}[(1-\lambda)-(1+\lambda) m] \\
\psi_{0}(\xi)= & \frac{P c}{2 \xi}[(1-\lambda)-(1+\lambda) m] \frac{1+m \xi^{2}}{\xi^{2}-m} \\
& +\frac{P c}{2 \xi}[(1+\lambda)-(1-\lambda) m] .
\end{aligned}
$$

The abovementioned formula and equating Formula (7) with Formula (8), the result will be

$$
\begin{aligned}
\varphi_{1}(\xi)= & \frac{P c(1+\lambda)}{4}\left(\xi+\frac{m}{\xi}\right) \\
& +\frac{P c}{2 \xi}[(1-\lambda)-(1+\lambda) m] \\
\psi_{1}(\xi)= & \frac{P c}{2 \xi}[(1-\lambda)-(1+\lambda) m] \frac{1+m \xi^{2}}{\xi^{2}-m} \\
& +\frac{P c}{2 \xi}[(1+\lambda)-(1-\lambda) m] \\
& +\frac{P c(1-\lambda)}{4}\left(\xi+\frac{m}{\xi}\right) .
\end{aligned}
$$

3.2. Stress Components of Elliptic Points. After obtaining the analytic functions $\varphi_{1}(\xi)$ and $\psi_{1}(\xi)$, finding the relationship between the various points of stress is required. Therefore, we first compute $\Phi(\xi)$ and $\Psi(\xi)$; that is,

$$
\begin{aligned}
\Phi(\xi) & =\frac{\varphi_{1}^{\prime}(\xi)}{\omega^{\prime}(\xi)} \\
& =\frac{P\left[(1+\lambda) \xi^{2}+(1+\lambda) m-2(1-\lambda)\right]}{4\left(\xi^{2}-m\right)} .
\end{aligned}
$$

Furthermore, equated to Formula (5), it will obtain

$$
\begin{aligned}
& \sigma_{\rho}+\sigma_{\theta}=4 \operatorname{Re}[\Phi(\xi)] \\
& =P \operatorname{Re}\left[\frac{\left[(1+\lambda) \xi^{2}+(1+\lambda) m-2(1-\lambda)\right]\left(e^{-2 i \theta}-m\right)}{\left(e^{2 i \theta}-m\right)\left(e^{-2 i \theta}-m\right)}\right] \\
& =P \frac{(1+\lambda)-(1+\lambda) m^{2}+2(1-\lambda) m}{1-2 m \cos 2 \theta+m^{2}} .
\end{aligned}
$$

The same can be concluded that

$$
\begin{aligned}
\Psi(\xi) & =\frac{\psi_{1}^{\prime}(\xi)}{\omega^{\prime}(\xi)}=P \\
& \cdot \frac{(1-\lambda) \xi^{2}+(1-\lambda) m-2(1+\lambda)+m^{2}(1+\lambda)}{4\left(\xi^{2}-m\right)} \\
& -\frac{P}{2}[(1-\lambda)-(1+\lambda) m]\left(1+m^{2}\right) \\
& \cdot\left[\frac{2 \xi^{2}}{\left(\xi^{2}-m\right)^{3}}+\frac{1}{\left(\xi^{2}-m\right)^{2}}+\frac{m}{\left(\xi^{2}-m\right)}\right] .
\end{aligned}
$$




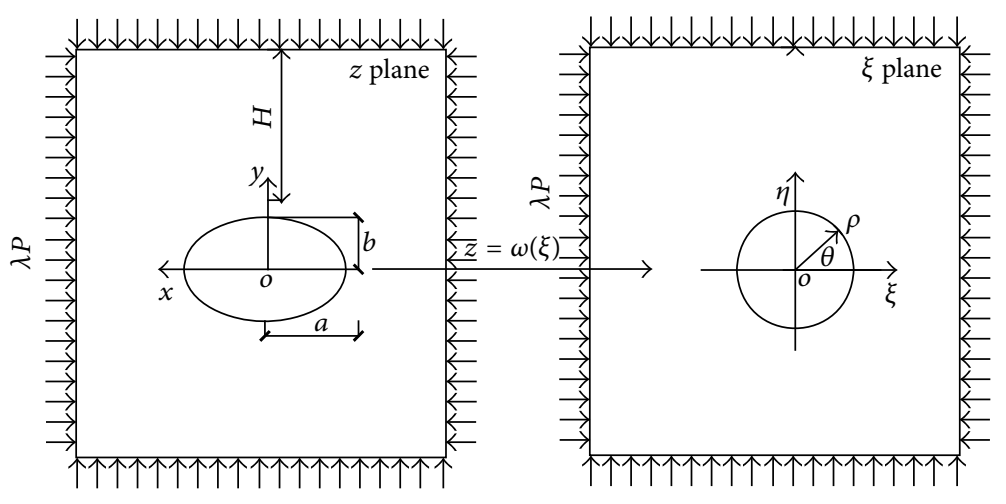

FIgURE 4: Conformal mapping area.

Equated to Formula (5), it can obtain

$$
\begin{aligned}
& \sigma_{\rho}-\sigma_{\theta}+2 i \tau_{\rho \theta}=\frac{2 \xi^{2}}{\rho^{2}\left(1-m \xi^{2}\right)}\left\{\frac{\left(1+m \xi^{2}\right)}{2}\left[-\frac{(1+\lambda) m-(1-\lambda)+\xi^{2}(1+\lambda)}{\left(\xi^{2}-m\right)^{2}}+\frac{(1+\lambda)}{\left(\xi^{2}-m\right)}\right]\right. \\
& +\frac{(1-\lambda) \xi^{2}+(1-\lambda) m-2(1+\lambda)+m^{2}(1+\lambda)}{4 \xi^{2}} \\
& \left.\quad-\frac{1}{2}[(1-\lambda)-(1+\lambda) m]\left(1+m^{2}\right)\left[\frac{2}{\left(\xi^{2}-m\right)^{2}}+\frac{1}{\xi^{2}\left(\xi^{2}-m\right)}+\frac{m}{\xi^{2}}\right]\right\} .
\end{aligned}
$$

3.3. Elliptical Tunnel Plastic and Plastic Zone Radius. When calculating the plastic district of the surrounding rock, the rock mass is assumed to be an ideal elastic-plastic model. Based on the elastic-plastic boundary, surrounding rock stress components relationship can be expressed by the abovementioned analytical solution and satisfy the rock breaking bad criteria using the modified Griffith failure criteria [23].

That is,

$$
\left(\sigma_{1}-\sigma_{3}\right)^{2}+k \sigma_{c}\left(\sigma_{1}+\sigma_{3}\right)=0,
$$

where $k$ is selected; namely, $0.5, \sigma_{c}$ is the maximum plastic radius values and $\sigma_{1}$ and $\sigma_{3}$ are the plane tunnels along two directions.

Equating Formulas (13) and (15) into Formula (16) will obtain $\rho_{\xi}$, and, taking $z=\omega(\xi)$ into the mapping function, you will obtain the plastic zone radius of the tunnel surrounding rock along the span and the hole depth direction in $z$ plane, $r_{x}$, and $r_{y}$. The application program was written using Fortran language to obtain the size and shape of the plastic zone.

\section{Analysis on Plastic Zone Deep Curved Wall of Horseshoe Tunnel}

The noncircular section tunnel problem commonly used in engineering problems included the horseshoe section tunnel, where an explicit expression is not specified. According to Formula (4), the mapping function and stress relationship problems are solved; that is, the problem is transformed into the solution of two parameters $R$ and $C_{k}$ in the mapping function [22]. Specific steps are as follows:

(1) Select the rectangular coordinate system in $z$ plane as shown in Figure 1. Take the tunnel axis of symmetry of the $x$ axis, which makes the horseshoe section tunnel distributed on both sides of the $x$-axis, and take point $i$ randomly around the tunnel on the left of the $x$-axis. Rectangular coordinates and corresponding polar coordinates in which the point corresponds are as follows: $\left(x_{i}, y_{i}\right)$ and $\left(\rho_{i}, \theta_{i}\right)$. When $i=0$, the corresponding coordinates are $\left(\rho_{0}, 0\right)$, and the corresponding right angle coordinates are $(1,0)$, and the polar coordinates in the plane $\xi$ on the unit circle; thus it will obtain

$$
R=\frac{r_{0}}{1+\sum_{k=0}^{\infty} C_{k}} .
$$

(2) To establish precision, the values of parameter $C_{k}$ are fixed using optimization techniques; the value of objective function is as follows:

$$
\begin{aligned}
f & =\sum_{j=0}^{m}\left\{r_{j}\right. \\
& \left.-R\left[\cos \left(\alpha_{j}-\beta_{j}\right)+\sum_{k=0}^{\infty} C_{k} \cos \left(\alpha_{j}+k \beta_{j}\right)\right]\right\}^{2} .
\end{aligned}
$$




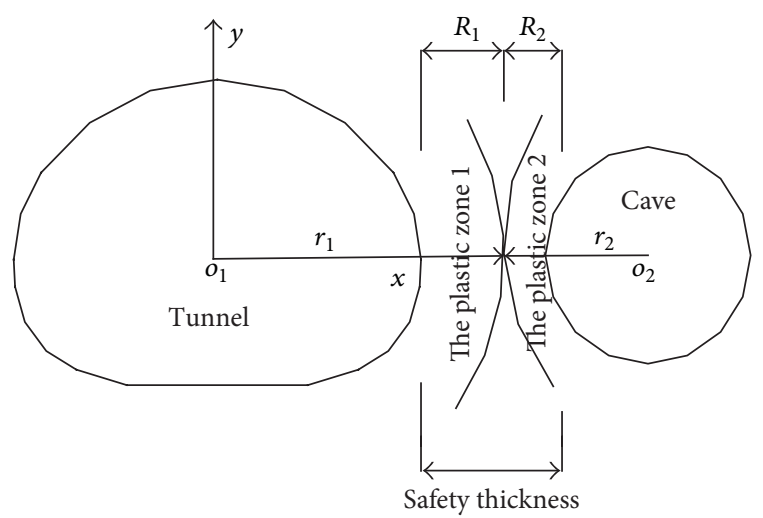

FIGURE 5: Mechanical calculation model of the critical safety of rock wall thickness.

In the above formula, $\rho_{i}$ and $\theta_{i}$ can be determined by points $C_{k}$ and $\beta_{i}$ as unknown quantity; then the minimum value of the objective function can be obtained when $f=0$. At this point, $C_{k}$ values are determined using the mapping function system.

After determining the value of $R$ and $C_{k}$, the relationship between the stress components according to Formulas (3), (4), and (5) can be obtained. The shape and size of plastic zone can be obtained by combining it with the Griffith force criterion.

\section{Determine the Thickness of Rock}

During the construction process, a certain thickness of the rock wall is typically retained to avoid the sudden collapse of the rock walls and to ensure the safety from accidents. During the design and construction of the tunnel in karst regions, scientific and effective identification of the location and size of karst tunnel and the critical thickness between the concealed karst caves and tunnels are issues that must be solved. When studying the critical safety distance between the tunnel and the rocks of karst cavern and determining the stability of rocks, the plastic district around the tunnels and caves serves as a standard for assessing the stability of the construction of the critical thickness analysis of medium and small dry caves and tunnels. The mechanics calculation mode of critical safe thickness between the caves and the rocks in the tunnels is shown in Figure 5.

According to the design standard of high-speed railway in China, the net effective area of double-lined railway tunnel is about 90-100 square meters, whereas the single-line railway tunnel is about $58-70$ square meters. The mountain tunnels are all closely related to oval and curved wall horseshoes. In this study, cave section is approximately round-shaped; the tunnel is approximately horseshoe-shaped tunnel.

Based on the analyses in Sections 3 and 4 and on the rock thickness mechanics model of the critical thickness of safety from Figure 3, the size of critical plastic zone in the side tunnels is described in Formula (19).

Guo and Qiao [19] indicate that when cave dimensions are small to facilitate the research, cave section shapes are typically considered as round, oval round, or nearly circular. Based on the analysis, the size of the tunnel cave is described in Formula (20).

The middle and small concealed karst caves located in the lateral part of the tunnel should meet the incoherence of the plastic zones of plastic zone of the tunnel cave and the cave tunnel to guarantee the cave stability between the tunnel and the rock wall. Therefore, the critical thickness of rock between the hidden caves and tunnels is described in Formula (21).

$$
\begin{aligned}
& H_{1}=r_{1}-\frac{d}{2} \\
& H_{2}=r_{2}-e \\
& H \geq r_{1}-\frac{d}{2}+r_{2}-e .
\end{aligned}
$$

In the above formula, $H_{1}$ stands for the size of cave side tunnels. $r_{1}$ represents the plastic zone size of corresponding different corners of the tunnel, that is, the radius in the plastic zone above the corresponding different angles; $d$ stands for the span of the cave of the side tunnels. Among all of them, $e$ stands for the studied plastic zone size of the cave, $r_{2}$ represents the plastic zone size of corresponding different corners of the tunnel, and $e$ stands for the shaft length of the round surface.

Similarly, when a cave is at the top or bottom of the tunnel, the ratio of the depth to the diameter is relatively large in deep-buried tunnels. Therefore, considering the effect of gravity gradient for analysis is unnecessary. The calculation is consistent with the public type side of the cave. The only difference depends on the level of angles and the corresponding polar radius (the corresponding value $\theta$ is different when calculating $r_{1}$ and $r_{2}$ ).

\section{Engineering Example Analysis and Model Validation}

The Doumo tunnel of the newly built Shanghai-Kunming rail (Guizhou segment) is located between the Anshun western station and Guanling station of Guizhou segment of the railway from Changchun to Kunming. The tunnel is a passenger-dedicated double track tunnel with the designed speed of $350 \mathrm{~km}$ per hour. The tunnel import mileage is D1K $841+144$, export mileage is D1K $843+220$, the length is 2076 , and the design adopted a Y-shaped slope. The rock formations that the hole gets through are mainly two lower Permian Maokou formation (P1m) limestones and middle Permian Longtan formation (P2L) clip mudstone, shale sandstone, limestone, and coal seams, and Upper Permian Changxing formation of Permian Dalong-Group (P2c+d) mudstone, sandstone, marl clip coal, lower Triassic Yelang group (Tly) clay stone, siltstone, limestone, and shale limestone, group of lower Triassic yongning (Tlyn1) limestone, dolomitic limestone clip mudstones, marls and lower Triassic Yongning second segment (Tlyn2) dolostone, argillaceous dolostone, argillaceous white, and dolomite mud limestone. The site tectonic denudates landforms in the lower mountains with a maximum depth of about $250 \mathrm{~m}$ above the vaults. The station 


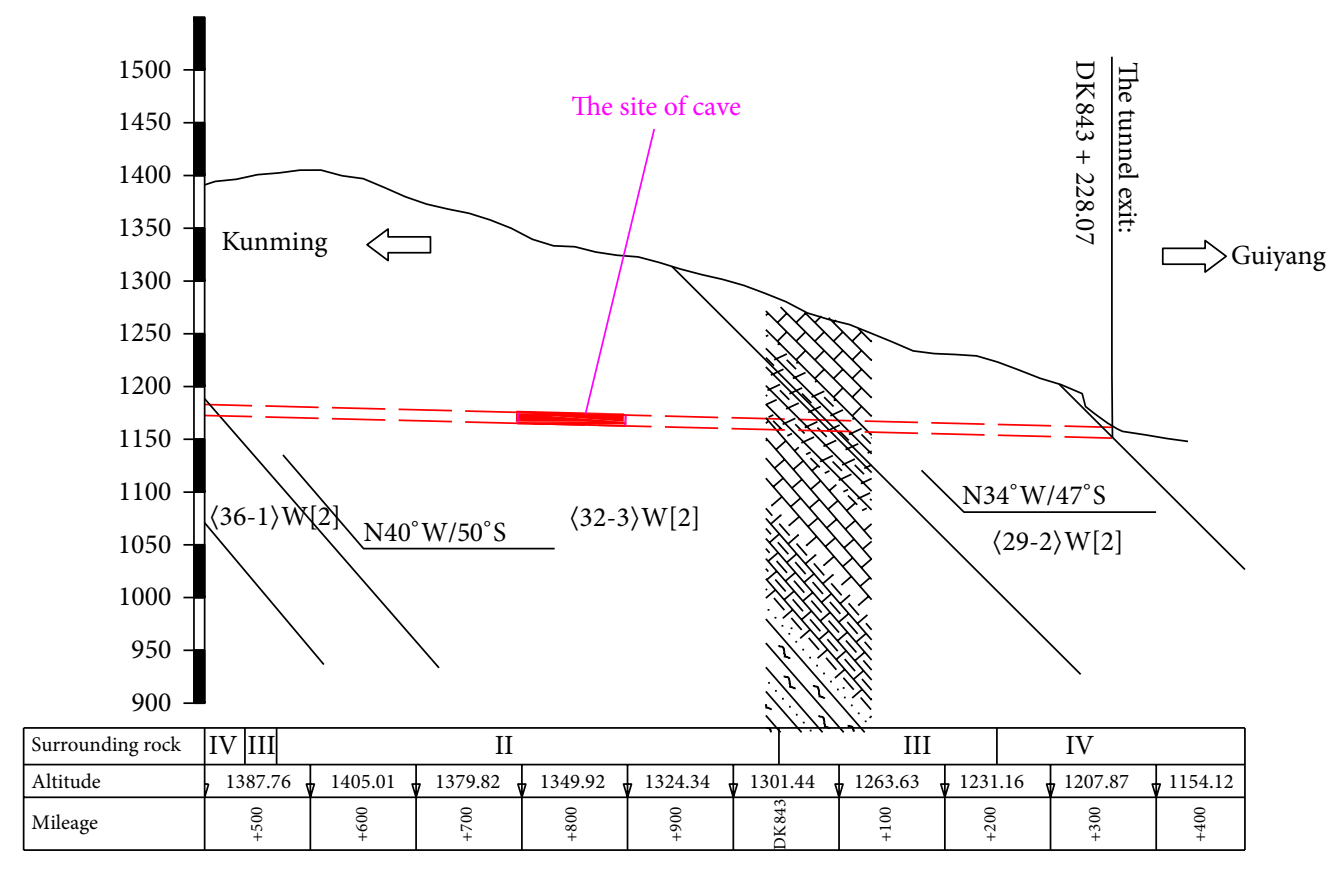

Figure 6: Topographic map of the Doumo tunnel.

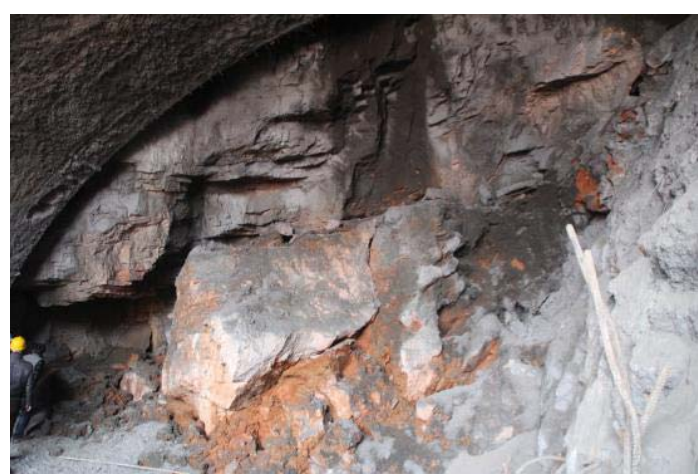

FIGURE 7: The tunnel surrounding rock of cave site.

and the topographic map of the Doumo tunnel is shown in Figure 6.

The tunnel excavation to mileage segment-D1K843+ $100 \sim 120 \mathrm{~m}$ found a cave located on the right side of the tunnel route through advanced detection. The cave is positioned to the right-side wall karst tunnel development pipeline, and the diameter is $9.45 \mathrm{~m}$. The tunnel surrounding rock of cave site in Figure 7 and the positional relationship of the tunnel and the cave is shown in Figure 8.

6.1. Numerical Calculation of Critical Safety Thickness. Based on the project, this paper uses an ABAQUS software to analyze the cave location. Shear and tensile failures are considered in the elastic-plastic numerical analysis. To observe the range and size of the failure zone in the numerical analysis, we determine the failure zone using the following steps: (a) Observe whether the tapped line or intersection exits in the active damage area of the two consecutive surfaces. If the answer is yes, the failure mechanism works and failure occurs.

(b) Observe the displacement velocity vector diagram. If the displacement velocity vector diagram presents movement consistent with the failure mechanism, the judgment of the first step is confirmed and the failure is happening.

(c) If the tapped line or intersection does not exist in the active damage zone, compare the first and the latter graphics after performing several steps. If the damage area is small, it indicates that the system is developing to the direction of possible equilibrium; if there is an increase and only the velocity vector can provide evidence, the system loses stability and suffers damage.

Establish the numerical analysis model in accordance with the above decision instability steps. The model size is selected as square three-dimensional rock mass modulus by $120 \mathrm{~m} \times 60 \mathrm{~m} \times 40 \mathrm{~m}$ and the vertical height from the top of the model to the cavity center is $60 \mathrm{~m}$. Based on the conditions of cavity depth, the upper rock mass weight model will be converted into uniform load to be applied on the model boundary. The bottom of the model applies the displacement boundary condition, and the left and right sides of the model apply the boundary stress on the rock side and the upper part is a free boundary. Six processes are used for excavation. In the calculation model, the height of the tunnel is $10 \mathrm{~m}$. The physical and mechanical parameters of the surrounding rock are listed in Table 1. 


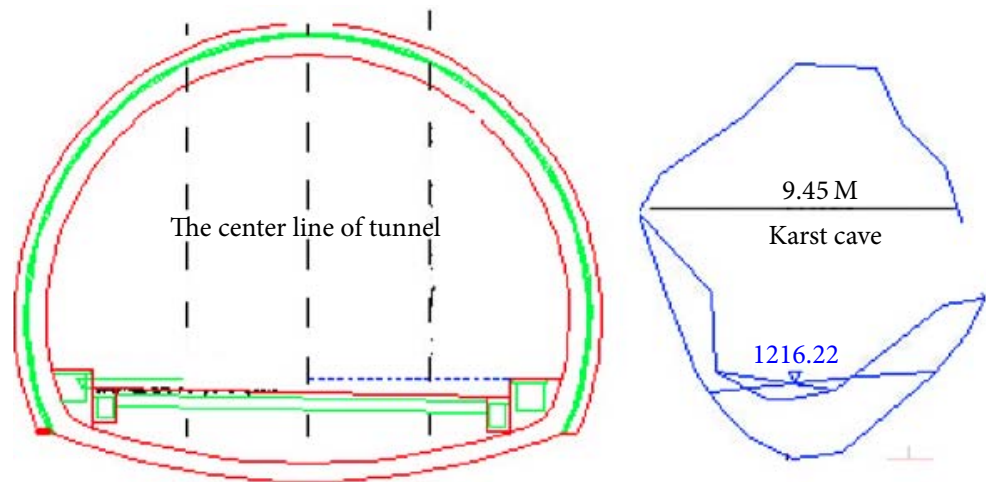

Figure 8: Positional relationship of tunnel and cave.

TABLE 1: Physical and mechanical parameters of the surrounding rock.

\begin{tabular}{lccccc}
\hline $\begin{array}{l}\text { Density } \\
\left(\mathrm{kg} / \mathrm{m}^{3}\right)\end{array}$ & $\begin{array}{c}\text { Elastic modulus } \\
(\mathrm{GPa})\end{array}$ & $\begin{array}{c}\text { Poisson's } \\
\text { ratio } \\
\mu\end{array}$ & $\begin{array}{c}\text { Material cohesive force } \\
(\mathrm{MPa})\end{array}$ & $\begin{array}{c}\text { Material frictional angle } \\
\left({ }^{\circ}\right)\end{array}$ & Permeability coefficient \\
\hline 2550 & 2.1 & 0.25 & 0.2 & 53 & $2 \times 10^{-7}$ \\
\hline
\end{tabular}

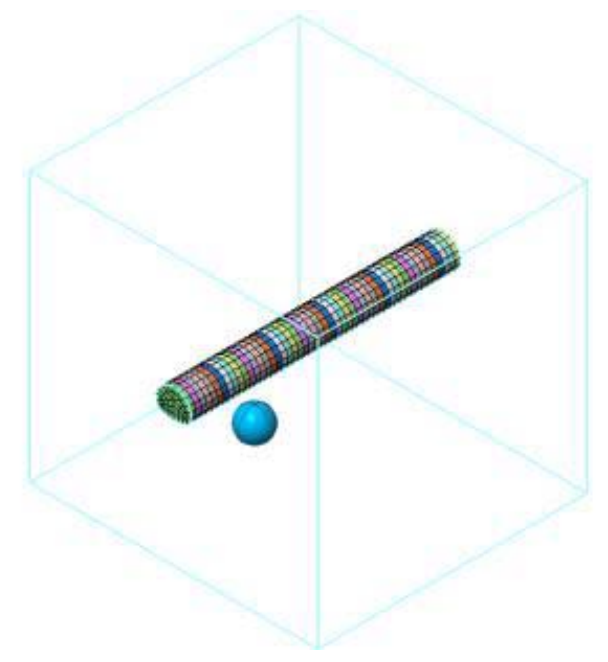

FIGURE 9: The three-dimensional calculation model.

In the numerical simulation analysis, the bearing capacity of argillaceous limestone used in the test is $200 \mathrm{kPa}$. The rock thickness analysis, that is, the analysis of the surrounding rock stress, exceeds $800 \mathrm{kPa}$ in the dissolved region between the cavity and the tunnel. The two-step method for excavation is employed, and the axial distance between the upper and lower steps along the tunnel is $3 \mathrm{~m}$. In accordance with the excavation simulation scene construction orders, as well as the Mises Guidelines, the surrounding rock stress is specified. In the following surrounding rock stress nephogram, the areas with a stress over $800 \mathrm{kPa}$ area are shown in gray. The three-dimensional calculation model is shown in Figures 9 and 10.

From Figures 7 and 8, in each tunnel excavation process, the maximum surrounding rock pressure of up and down
TABLE 2: Maximum stress after the excavation stability at every step.

\begin{tabular}{lccc}
\hline Step & $\begin{array}{c}\text { Stress } \\
(\mathrm{Mpa})\end{array}$ & Step & $\begin{array}{c}\text { Stress } \\
(\mathrm{Mpa})\end{array}$ \\
\hline The first up-step & 1.21 & The first down-step & 1.53 \\
The second up-step & 1.69 & The second down-step & 1.99 \\
The third up-step & 1.94 & The third down-step & 2.32 \\
The forth up-step & 2.48 & The forth down-step & 2.87 \\
The fifth up-step & 2.79 & The fifth down-step & 2.94 \\
The sixth up-step & 2.64 & - & - \\
\hline
\end{tabular}

steps as shown in Figure 11, the maximum value of stress after the stability of the surrounding rock in each process is shown in Table 2.

Based on Formulas (12)-(14), the karst cave and the tunnel went through in a high stress area at this time and easily directed to the instability of the surrounding. According to the numerical model analysis, the safety rock thickness of this cave is $3.5 \mathrm{~m}$.

6.2. Analysis of the Critical Safety Thickness according to the Plastic Zone Model. Critical safety thickness is searched according to the profile of the cave on the basic forms and the position relationship between tunnels and caves by using Formulas (19), (20), and (21).

The critical safety thickness of rock is calculated according to Formulas (19), (20), and (21) as follows:

$$
H \geq r_{1}-\frac{d}{2}+r_{2}-e \geq 4.63 \mathrm{~m} .
$$

6.3. Contrast with Actual Excavation. In the actual engineering construction, a minimum rock thickness of about $4.46 \mathrm{~m}$ is used, which is larger than the calculated thickness by the 


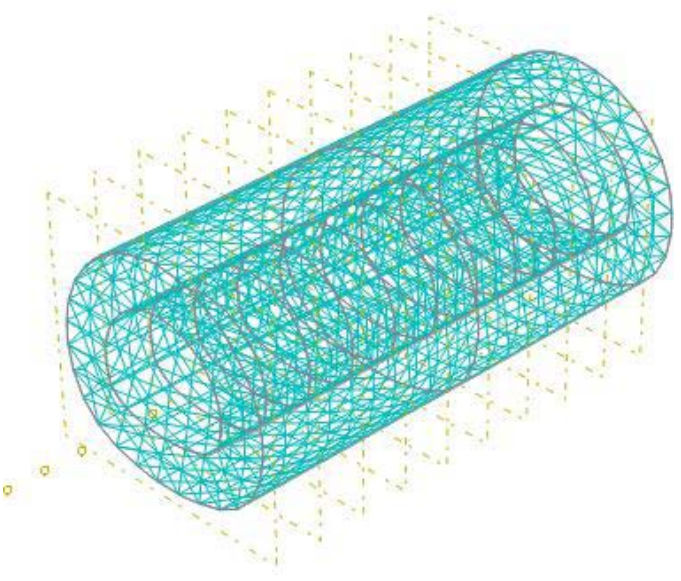

FIGURE 10: Mesh generation of tunnel.

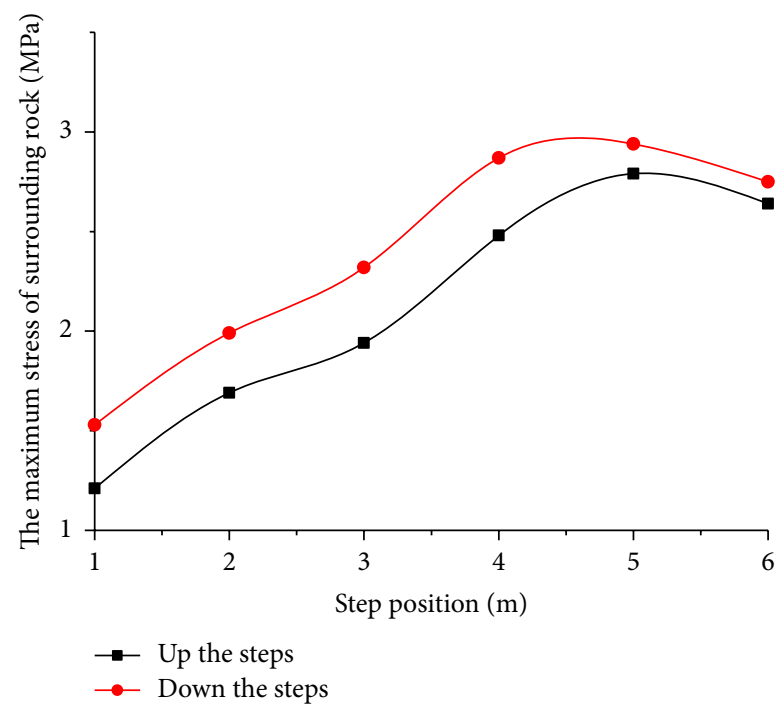

FIGURE 11: Maximum surrounding rock pressure of up and down steps.

plastic zone model and numerical analysis of the structure. In the cave section, steel frame of model 122a and concrete arch protection with C30 with a thickness of $0.5 \mathrm{~m}$ are used and a thick dry rubble buffer layer with a depth of $1.5 \mathrm{~m}$ is established. Two $200 \mathrm{~mm}$ diameter vertical drainage hoses are initially embedded inside the cave and connected with the arch foot and blind ditch.

\section{Conclusion}

The article combines the conformal mapping function by Verruijt and the complex variable function method by Muskhelishvili, using the Fortran language programming process application to deduce the formula for calculating the size and scope of the oval tunnel and the horseshoeshaped tunnel of the curved wall of the tunnel. The the plastic zone calculation is applied to the calculation of critical safety thickness of rocks between the medium and small dry cave and the tunnel project. The calculating model analysis of caves in different shapes and different ranges is established, as well as the plastic zone shape and the run through of plastic zone. To verify its feasibility and rationality based on a certain cave of Doumo tunnel of new Shanghai-Kunming high-speed railway, the numerical model to analyze and compare is built and connected with the excavation of the engineering process. By comparing the two models, we obtain the following conclusions:

(1) Establish mechanical calculation mode of the rock thickness of medium small dry caves and tunnels under different space conditions based on the conformal mapping function and the complex variable function method of plastic areas.

(2) Compare the result of rock critical safety thickness with the complex variable function method with the actual engineering process. The process is approximately an analytical method. With few parameters being used, it is easy to get numerical results, and the results are very close to the actual engineering process, which are reliable and rational. Therefore, the results can serve as a reference guide for this kind of engineering process.

\section{Conflicts of Interest}

No conflicts of interest exist regarding the publication of this manuscript.

\section{Acknowledgments}

This work is supported by the National Natural Science Foundation of China (Grants nos. 41372351 and 41672339).

\section{References}

[1] H. Kastner, Tunnel and Tunnel Statics, Shanghai Science and Technology Press, 1980, Translated by Tongji University.

[2] G. D. Donald and F. M. Samir, "Linear source in a circular tunnel," IEEE Transactions on Antennas and Propagation, vol. 54, no. 7, pp. 2034-2047, 1980.

[3] X. F. Yu and Y. R. Zheng, Rock stability analysis of underground engineering, Beijing Coal Industry Press, 1980.

[4] J. H. Wang, J. B. Zhong, and Z. J. Chen, Rock and Soil Mechanics, vol. 21, no. 3, pp. 201-204, 2009.

[5] Y. Pan and Z.-Q. Wang, "Elasto-plastic analysis on surrounding rock of circular chamber based on strain nonlinear engineering," Journal of Rock Mechanics and Engineering, vol. 24, no. 6, pp. 915-920, 2009.

[6] B. Behnam, S. Fazlollah, and M. Hamid, "Prediction of plastic zone size around circular tunnels in non-hydrostatic stress field," International Journal of Mining Science and Technology, vol. 24, no. 1, pp. 81-85, 2014.

[7] L.-W. Chen, J.-B. Peng, W. Fan, and P. Sun, "Analysis of surrounding rock mass plastic zone of round tunnel under non-uniform stress field based on the unified strength theory," Journal of China Coal Society, vol. 32, no. 1, pp. 20-23, 2007. 
[8] J. S. Sun and W. B. Lu, "Analytical elastic-plastic solutions to supporting rock masses of circular tunnels under asymmetric load," Rock and Soil Mechanics, vol. 20 Supplement, pp. 327-332, 2007.

[9] G. E. Exadaktylos, P. A. Liolios, and M. C. Stavropoulou, "A semi-analytical elastic stress-displacement solution for notched circular openings in rocks," International Journal of Solids and Structures, vol. 40, no. 5, pp. 1165-1187, 2003.

[10] X. R. Liu, M. Huang, and W. H. Zhu, "The radius of the plastic zone of deep circular tunnel under a supporting anchor," Journal of Chongqing University, pp. 573-576, 2008.

[11] A. Verruijt, "A complex variable solution for a deforming circular tunnel in an elastic half-plane," International Journal for Numerical and Analytical Methods in Geomechanics, vol. 21, no. 2, pp. 77-89, 1997.

[12] E. H. Zha, X. Z. Hua, and D. H. Chen, "Quantitative analysis of plastic region in deep buried tunnel based on in-situ stress test," Journal of Experimental Mechanics, vol. 28, no. 5, pp. 657-666, 2013.

[13] S. A. Massinas and M. G. Sakellariou, "Closed-form solution for plastic zone formation around a circular tunnel in half-space obeying Mohr-Coulomb criterion," Geotechnique, vol. 59, no. 8, pp. 691-701, 2009.

[14] F. Chen, D. Zhang, and S. Zhang, "Solving the mapping function of noncircular underground cavern with the method of successive approximation," China Railway Science, vol. 34, no. 2, pp. 80-86, 2013.

[15] Z. Gao, X. Meng, and G. Wang, "Analysis of the plastic zone of surrounding rock under seepage and a non-axisymmetric load," Modern Tunnelling Technology, vol. 52, no. 2, pp. 70-75, 2014.

[16] A. R. Kargar, R. Rahmannejad, and M. A. Hajabasi, "A semianalytical elastic solution for stress field of lined non-circular tunnels at great depth using complex variable method," International Journal of Solids and Structures, vol. 51, no. 6, pp. 14751482, 2014.

[17] C. Q. Liu and H. J. Peng, "Analysis of the safe distance between a tunnel face and karst cave," Modern Tunneling Technology, vol. 49, no. 3, pp. 109-113, 2012.

[18] Y. L. Zhao, Q. H. Wu, and W. J. Wang, "Strength reduction method to study stability of goaf overlapping roof based on catastrophe theory," Chinese Journal of Rock Mechanics and Engineering, vol. 29, no. 7, pp. 1424-1434, 2010.

[19] J. Q. Guo and C. S. Qiao, "Plastic zone around elliptical hole portal and its application in karst tunnel," China Railway Science, vol. 35, no. 3, pp. 108-113, 2013.

[20] N. I. Muskhelishvili and J. R. M. Radok, Some basic problems of the mathematical theory of elasticity, Cambridge University Press, Cambridge, UK, 1953.

[21] Y. Z. Shi and X. N. Gao, "Complex variable function solution of stress and displacement of surrounding rock buried deep horseshoe-shaped tunnel excavation," Journal of Civil, Architectural and Environmental Engineering, vol. 36, no. 1, pp. 101-105, 2014.

[22] A. Z. Lv and L. Q. Zhang, Underground tunnel complex variable method of mechanical Analysis, Science press, 2007.

[23] E. Hoek and Z. T. Bieniawski, "Brittle fracture propagation in rock under compression," International Journal of Fracture Mechanics, vol. 26, no. 4, pp. 276-294, 1984. 


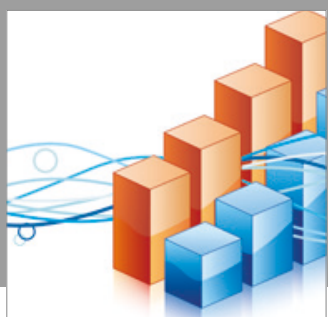

Advances in

Operations Research

vatersals

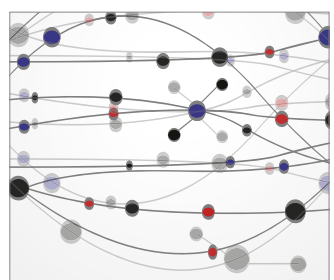

\section{The Scientific} World Journal
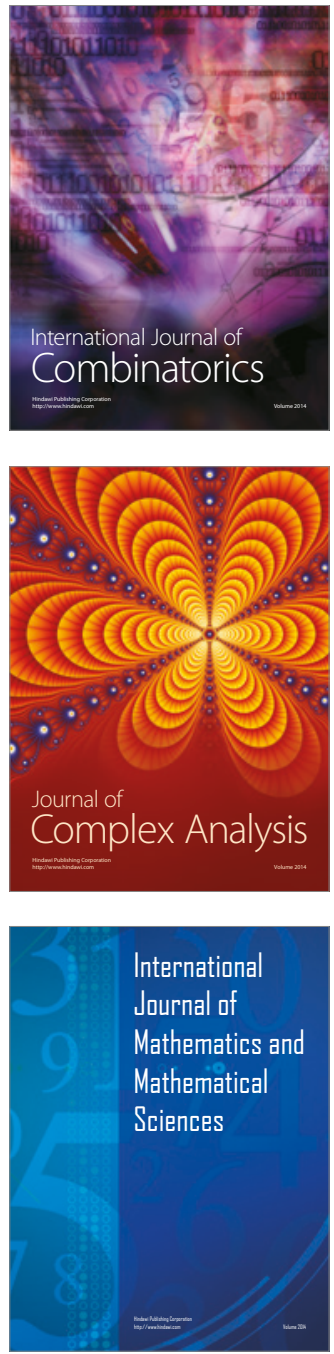
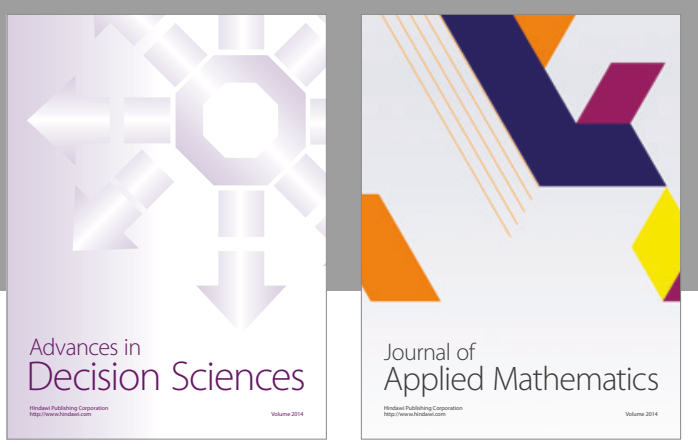

Algebra

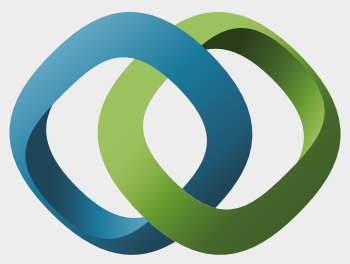

\section{Hindawi}

Submit your manuscripts at

https://www.hindawi.com
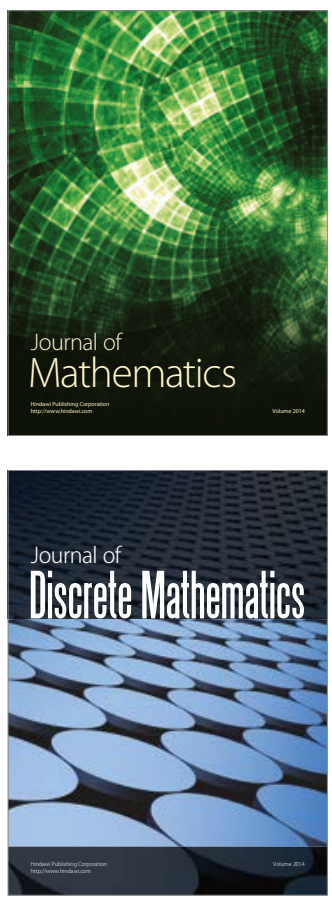

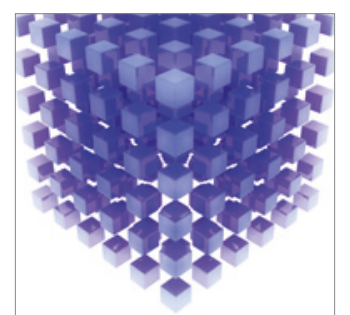

Mathematical Problems in Engineering
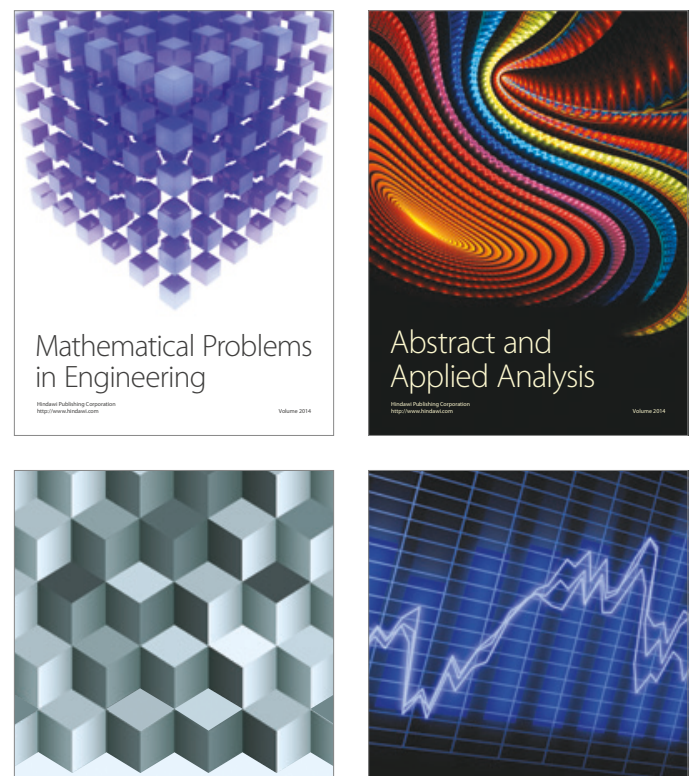

Journal of

Function Spaces

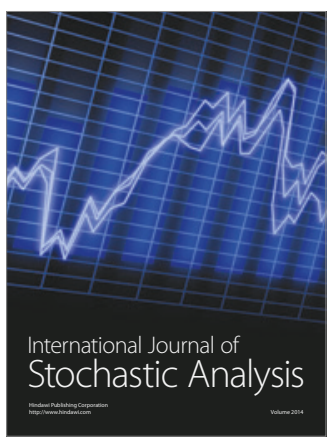

Probability and Statistics
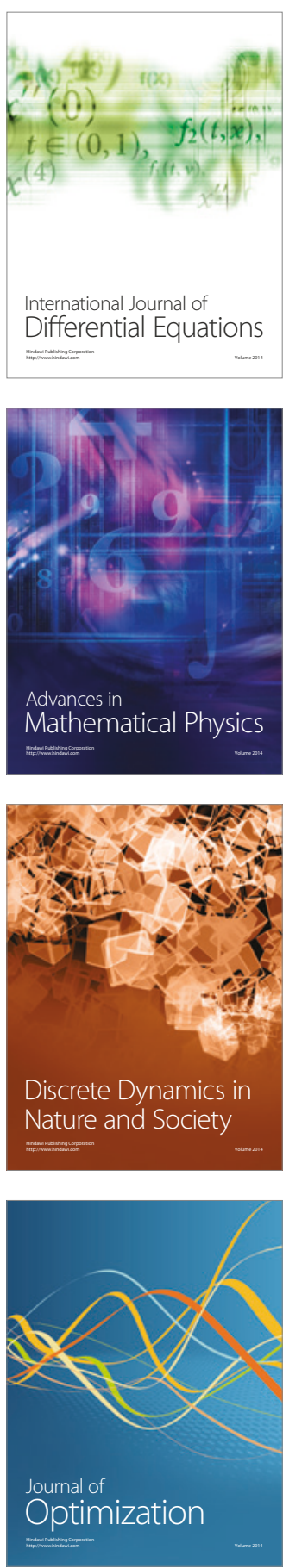